\title{
Genetic Predisposition to the Metabolism of Irinotecan (CPT-11) Role of Uridine Diphosphate Glucuronosyltransferase Isoform 1A1 in the Glucuronidation of its Active Metabolite (SN-38) in Human Liver Microsomes
}

\author{
Lalitha lyer, ${ }^{\star}$ Christopher D. King," Peter F. Whitington,§ Mitchell D. Green, " Sandip K. Roy, ${ }^{\ddagger}$ Thomas R. Tephly, \\ Birgit L. Coffman," and Mark J. Ratain ${ }^{\star * \|}$ \\ $*$ Committee on Clinical Pharmacology, ${ }^{\ddagger}$ Department of Medicine, ${ }^{\S}$ Department of Pediatrics, and $\|_{\text {Cancer Research Center, The }}$ \\ University of Chicago, Chicago, Illinois 60637; and ${ }^{\mathbb{I}}$ Department of Pharmacology, The University of Iowa, Iowa City, Iowa 52242
}

\begin{abstract}
Irinotecan (CPT-11) is a promising antitumor agent, recently approved for use in patients with metastatic colorectal cancer. Its active metabolite, $\mathrm{SN}-38$, is glucuronidated by hepatic uridine diphosphate glucuronosyltransferases (UGTs). The major dose-limiting toxicity of irinotecan therapy is diarrhea, which is believed to be secondary to the biliary excretion of $\mathrm{SN}-38$, the extent of which is determined by $\mathrm{SN}-38$ glucuronidation. The purpose of this study was to identify the specific isoform of UGT involved in SN-38 glucuronidation. In vitro glucuronidation of $\mathrm{SN}-38$ was screened in hepatic microsomes from normal rats $(n=4)$, normal humans $(n=25)$, Gunn rats $(n=3)$, and patients $(n=4)$ with Crigler-Najjar type I (CN-I) syndrome. A wide intersubject variability in in vitro $\mathrm{SN}-38$ glucuronide formation rates was found in humans. Gunn rats and CN-I patients lacked SN-38 glucuronidating activity, indicating the role of UGT1 isoform in $\mathrm{SN}-38$ glucuronidation. A significant correlation was observed between $\mathrm{SN}-38$ and bilirubin glucuronidation $(r=0.89 ; P=0.001)$, whereas there was a poor relationship between para-nitrophenol and $\mathrm{SN}-38$ glucuronidation $(r=$ $0.08 ; P=0.703$ ). Intact $\mathrm{SN}-38$ glucuronidation was observed only in HK293 cells transfected with the UGT1A1 isozyme. These results demonstrate that UGT1A1 is the isoform responsible for $\mathrm{SN}-38$ glucuronidation. These findings indicate a genetic predisposition to the metabolism of irinotecan, suggesting that patients with low UGT1A1 activity, such as those with Gilbert's syndrome, may be at an increased risk for irinotecan toxicity. (J. Clin. Invest. 101:847854.) Key words: glucuronosyltransferase - irinotecan Crigler-Najjar syndrome • Gilbert's syndrome • Gunn rats
\end{abstract}

Preliminary reports of this study were presented at the American Society of Clinical Pharmacology and Therapeutics meeting in March 1997, and at the American Society of Clinical Oncology meeeting in May 1997.

Address correspondence to Mark J. Ratain, M.D., The University of Chicago, 5841 South Maryland Avenue, MC 2115, Chicago, IL 60637. Phone: 773-702-4400; FAX: 773-702-0963; E-mail: mjratain@ mcis.bsd.uchicago.edu

Received for publication 13 June 1997 and accepted in revised form 8 December 1997.

J. Clin. Invest.

(C) The American Society for Clinical Investigation, Inc. 0021-9738/98/02/0847/08 \$2.00

Volume 101, Number 4, February 1998, 847-854

http://www.jci.org

\section{Introduction}

Irinotecan (CPT-11) $)^{1}$ is a semisynthetic analogue of the cytotoxic alkaloid camptothecin (CPT), which is obtained from the oriental tree, Camptotheca acuminata. Irinotecan \{7-ethyl-10[4-(1-piperidino)-1-piperidino]carbonyloxycamptothecin] is an inhibitor of topoisomerase I enzyme and has exhibited promising antitumor activity in various experimental tumors (1-3) and in colorectal cancer patients (4). Recently, it has been approved (Camptosar ${ }^{\circledR}$, Pharmacia \& Upjohn, Inc., Windsor, Berkshire, UK) in the United States for the treatment of metastatic colorectal cancer refractory to 5-fluorouracil therapy.

Irinotecan is biotransformed (Fig. 1) by tissue and serum carboxylesterases to an active metabolite, SN-38 (7-ethyl10-hydroxycamptothecin) $(5,6)$, which has a 100-1,000-fold higher antitumor activity than irinotecan. In addition, irinotecan has been shown recently to be metabolized by hepatic cytochrome P-450 3A enzymes to 7-ethyl-10-[4- $N$-(5-aminopentanoic acid)-1-piperidino] carbonyloxycamptothecin (APC), which has 500-fold weaker antitumor activity compared with SN-38 $(7,8)$. SN-38 undergoes significant biliary excretion and enterohepatic circulation (9). It is subjected to glucuronidation by hepatic uridine diphosphate glucuronosyltransferases (UGTs) to form SN-38 glucuronide (10-O-glucuronyl-SN-38, $\mathrm{SN}-38 \mathrm{G})$, which is inactive and excreted into the bile and urine. SN-38G can be deconjugated to form SN-38 by intestinal $\beta$-glucuronidase enzyme (10).

The major dose-limiting toxicities of irinotecan include diarrhea and, to a lesser extent, myelosuppression. Incidences of irinotecan-induced diarrhea can be serious and do not respond adequately to conventional antidiarrheal agents (11). This diarrhea is due possibly to direct enteric injury caused by the active metabolite, $\mathrm{SN}-38$, which has been shown to accumulate in the intestine after intraperitoneal administration of irinotecan in athymic mice (12). In a recently completed phase I clinical trial, there was an inverse relationship between $\mathrm{SN}-38$ glucuronidation rates and severity of diarrheal incidences in patients treated with increasing doses of irinotecan (13). These findings indicate that glucuronidation of $\mathrm{SN}-38$ protects against irinotecan-induced gastrointestinal toxicity. Therefore, the conversion of SN-38 to SN-38G by hepatic UGTs is a criti-

\footnotetext{
1. Abbreviations used in this paper: APC, 7-ethyl-10-[4- $N$-(5-aminopentanoic acid)-1-piperidino] carbonyloxycamptothecin; AZT, zidovudine (3'-azido-3'-deoxythymidine); CN-I, Crigler-Najjar syndrome (type I); CPT, camptothecin; CPT-11, irinotecan; GAZT, glucuronide of AZT; $O^{6}$-BG, $O^{6}$-benzylguanine; OLPC, oleolyl lysophosphatidylcholine; PNP, para-nitrophenol; SN-38G, SN-38 glucuronide; UDPGA, uridine diphosphoglucuronic acid; UGT, uridine diphosphate glucuronosyltransferase.
} 


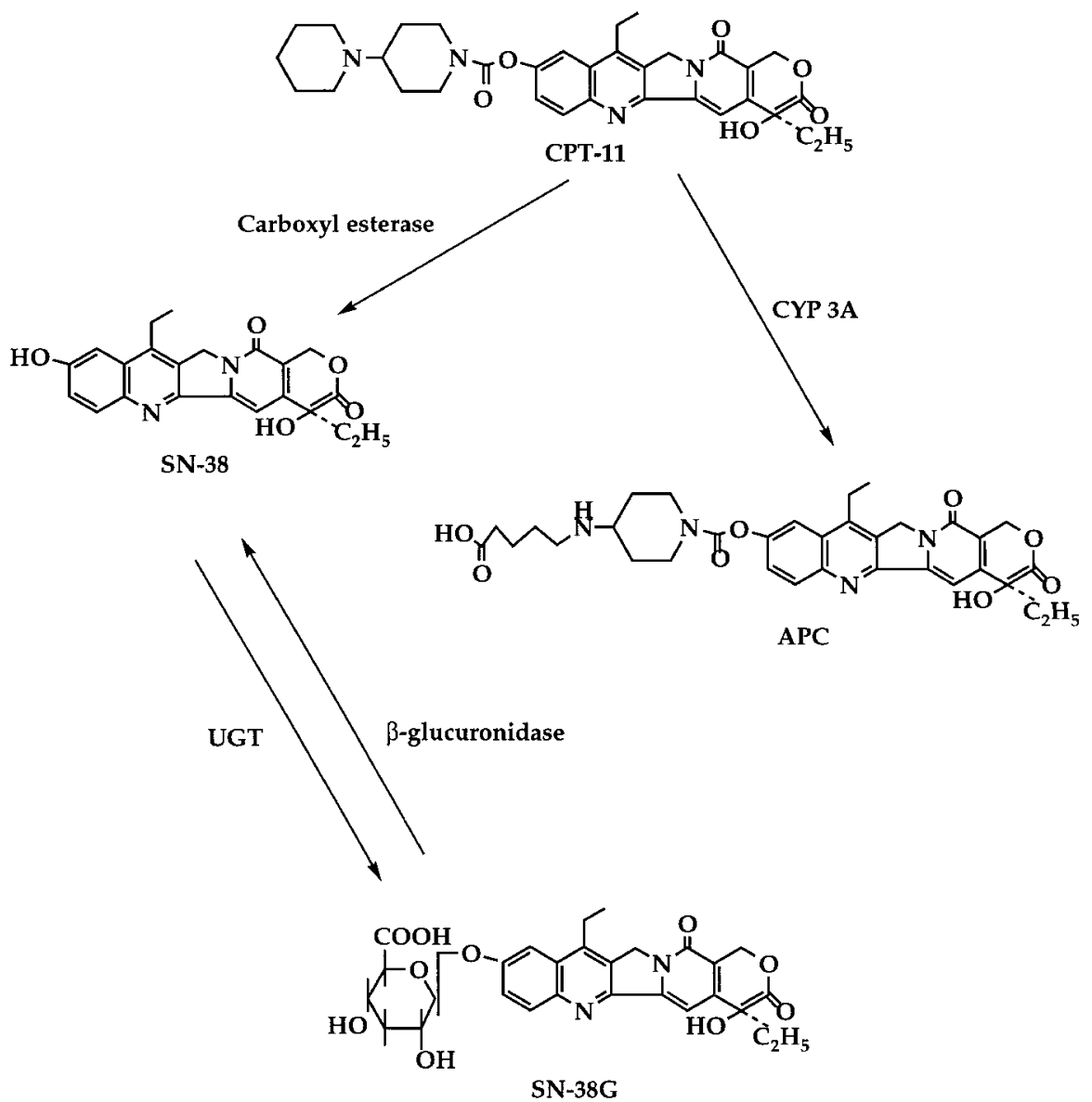

Figure 1. Metabolism of irinotecan to APC, SN-38, and SN-38G. cal step in the sequential metabolic pathway of irinotecan (Fig. 1). Differential rates of SN-38 glucuronidation among subjects may explain the considerable interindividual variation in the pharmacokinetic parameter estimates and toxicities observed after administration of irinotecan in cancer patients $(13,14)$.

Hepatic glucuronidation results from the activities of a multigene family of UGT enzymes, the members of which exhibit specificity for a variety of endogenous substrates and xenobiotics (15-17). The UGT enzymes are broadly classified into two distinct gene families. UGT1 is further subclassified into multiple isozymes, all of which have a conserved carboxyl region encoded by exons $2-5$, with a variable region encoded by various exons 1 . Isoforms of the UGT2 family are unique gene products of which at least eight isozymes have been identified (16-18).

The purpose of this study was to determine the UGT or UGTs responsible for SN-38 glucuronidation. To investigate this, we measured the in vitro metabolism of SN-38 by normal human and rat liver microsomes, as well as microsomes from Gunn rats $(19,20)$ and patients with Crigler-Najjar type I syndrome (CN-I) $(21,22)$, both of which lack UGT1 activity. SN-38 glucuronidation was correlated with glucuronidation of other substrates (bilirubin and para-nitrophenol [PNP]) known to be glucuronidated by specific isoforms of UGT. In addition, human embryonic kidney cells (HK293) expressing specific isoforms of UGT were evaluated for their reactivity with SN-38.

\section{Methods}

Liver microsomes were prepared from normal humans $(n=25)$, CN-I patients $(n=4)$, normal rats $(n=3$, Sprague-Dawley; Harlan Sprague Dawley, Inc., Indianapolis, IN), and Gunn rats $(n=3$, Harlan Sprague Dawley, Inc.). Microsomes from human liver samples $(n=$ 10) medically unsuitable for liver transplantation were acquired under the auspices of the Washington Regional Transplant Consortium (Washington, DC). In addition, microsomes $(n=15)$ were prepared by differential centrifugation methods (23), using human liver samples obtained with the approval of the Institutional Review Boards of institutions involved through the Liver Tissue Procurement and Distribution System (National Institutes of Diabetes, Digestive and Kidney Diseases NO1-DK6-2274). Rat killing and surgical removal of liver lobes were performed in accordance with the guidelines of the Institutional Animal Care and Use Committee of the University of Chicago. The various investigations were performed in pooled microsomes from normal rats $(n=4)$, normal humans $(n=10)$, and Gunn rats $(n=3)$, while microsomes from CN-I patients were used individually. Total protein content of microsomes was determined by the method of Bradford (24).

SN-38, SN-38G, and CPT were gifts kindly provided by Dr. Kiyoshi Terada (Yakult Honsha Co., Ltd., Japan). $O^{6}$-benzylguanine $\left(O^{6}\right.$-BG) and $O^{6}$-benzyl-8-oxoguanine were gifts kindly provided by Dr. Robert Moschel (Carcinogen-modified Nucleic Acid Chemistry, ABL-Basic Research program, National Cancer Institute-Frederick Cancer Research and Development Center). Radiolabeled uridine diphosphoglucuronic acid ([ $\left.{ }^{14} \mathrm{C}\right]$ UDPGA) was obtained from ICN Biomedicals Inc. (Irvine, CA). UDPGA, $\beta$-glucuronidase, $3^{\prime}$-azido-3'- 
deoxythymidine (AZT), glucuronide of AZT (GAZT), BSA, magnesium chloride $\left(\mathrm{MgCl}_{2}\right)$, PNP, ethylenediamine tetraacetic acid, glycine, trizma [Tris(hydroxymethyl)aminomethane] base, saccharolactone (D-saccharic acid 1,4-lactone), L- $\alpha$-phosphatidylcholine, oleolyl lysophosphatidylcholine (OLPC), NAD 3'-phosphate reduced form ( $\beta$ NADPH), bilirubin, and Triton X-100 were all obtained from Sigma Chemical Co. (St. Louis, MO). Ammonium formate, acetonitrile, phosphoric acid, ammonium hydroxide, potassium dihydrogen phosphate $\left(\mathrm{KH}_{2} \mathrm{PO}_{4}\right)$, ethyl acetate, and methanol were obtained from Fisher Scientific Co. (Fairlawn, NJ).

$S N-38$ glucuronidation assay. Microsomes $(1 \mathrm{mg} / \mathrm{ml})$ were incubated with SN-38 $(5 \mu \mathrm{M}), \mathrm{MgCl}_{2}(10 \mathrm{mM})$, and UDPGA $(5 \mathrm{mM})$ in a $0.1 \mathrm{M}$ Tris- $\mathrm{HCl}$ buffer medium (pH 7.4) for $30 \mathrm{~min}$ at $37^{\circ} \mathrm{C}$, in a final volume of $200 \mu \mathrm{l}(25)$. After stopping the reaction with $400 \mu \mathrm{l}$ methanol, the incubation mix was centrifuged at $2,500 \mathrm{rpm}$ for $30 \mathrm{~min}$ at $4^{\circ} \mathrm{C}$. The supernatant was evaporated to dryness under nitrogen (Reacti-Therm ${ }^{\circledR}$ heating module; Pierce Chemical Co., Rockford, IL) and reconstituted with $250 \mu \mathrm{l}$ of mobile phase. $100 \mu \mathrm{l}$ aliquots were injected into a reverse phase HPLC assay system coupled to a fluorescence detector (Hitachi Instruments, Inc., San Jose, CA). CPT was used as the internal standard. The mobile phase consisted of $35 \%$ acetonitrile and $65 \% 0.1 \mathrm{M} \mathrm{KH}_{2} \mathrm{PO}_{4}$, containing $3 \mathrm{mM}$ sodium heptane sulphonate ( $\mathrm{pH} 4.0$ ), used at a flow rate of $0.8 \mathrm{ml} / \mathrm{min}$. $\mathrm{A} \mathrm{C}_{18} \mu$ Bondapak column $(10 \mu \mathrm{m}, 3.9 \times 300 \mathrm{~mm}$; Waters Corp., Milford, MA) was used with fluorescence detection at $355 \mathrm{~nm}\left(\lambda_{\mathrm{ex}}\right)$ and $515 \mathrm{~nm}\left(\lambda_{\mathrm{em}}\right)$.

The rates of formation of SN-38G were calculated using a standard curve of chromatographic peak height ratios of SN-38G to CPT. Reaction rates were shown to be linear for incubation times up to at least $1.5 \mathrm{~h}$, and for final microsomal protein concentrations of up to 2 $\mathrm{mg} / \mathrm{ml}(25)$. SN-38G formation was also measured in a human liver microsomal pool $(n=10)$ using a range of substrate concentrations to determine kinetic constants, $K_{\mathrm{m}}$ and $V_{\max }$, which were calculated using Lineweaver-Burk analysis (26).

AZT glucuronidation assay. This assay was modified from that described previously $(27,28)$. Microsomes were preincubated with OLPC $(0.8 \% \mathrm{wt} / \mathrm{wt}$, OLPC/microsomal protein $)$ at $4^{\circ} \mathrm{C}$ for $20 \mathrm{~min}$. The reaction mixture (final volume $300 \mu \mathrm{l})$ contained microsome (1 $\mathrm{mg} / \mathrm{ml}) / \mathrm{OLPC}$ mix, AZT $(5 \mathrm{mM}), \mathrm{MgCl}_{2}(10 \mathrm{mM}), 0.2 \mathrm{M}$ Tris- $\mathrm{HCl}$ buffer (pH 7.3), and UDPGA $(10 \mathrm{mM})$. After an incubation period of $1 \mathrm{~h}$ at $37^{\circ} \mathrm{C}$, the reaction was stopped with the addition of $30 \mu \mathrm{l}$ of $\mathrm{HCl}$. Proteins were removed after centrifugation at 10,500 $\mathrm{g}$ for $5 \mathrm{~min}$, and $30 \mu \mathrm{l}$ aliquots of the supernatant were injected into an HPLC system (Hitachi Instruments, Inc.) coupled to an ultraviolet detector $(\lambda=$ $265 \mathrm{~nm})$. The mobile phase consisted of a 15:85 mixture of acetonitrile/ammonium phosphate buffer $(0.1 \% \mathrm{vol} / \mathrm{vol}$ phosphoric acid, adjusted to $\mathrm{pH} 2.7$ with ammonium hydroxide) used at a flow rate of 1 $\mathrm{ml} / \mathrm{min}$.

$O^{6}-B G$ oxidation assay. Liver microsomes $(1 \mathrm{mg} / \mathrm{ml})$ were incubated with $O^{6}$-BG $(10 \mu \mathrm{M})$ in the presence of $\mathrm{MgCl}_{2}(50 \mathrm{mM})$ and $\beta$-NADPH $(1 \mathrm{mM})$ in $50 \mathrm{mM}$ potassium phosphate buffer $(\mathrm{pH} 7.4)$ at $37^{\circ} \mathrm{C}$ for $1 \mathrm{~h}$, in a final volume of $500 \mu \mathrm{l}(29)$. The reaction was terminated with $1 \mathrm{ml}$ chilled methanol $(100 \%)$. Samples were centrifuged, and supernatant was lyophilized and reconstituted in mobile phase for injection into an HPLC system coupled to a diode array detector (Hitachi Instruments, Inc.) $(\lambda=280 \mathrm{~nm})$. The mobile phase consisted of $35 \%$ methanol and $65 \% 50 \mathrm{mM}$ ammonium formate, $\mathrm{pH} 4.5$, used at a flow rate of $1.5 \mathrm{ml} / \mathrm{min}$.

Bilirubin glucuronidation assay. The formation of bilirubin monoand diglucuronides was quantitated using ${ }^{14} \mathrm{C}$-labeled UDPGA (30). Human liver microsomes $(0.5 \mathrm{mg} / \mathrm{ml})$ were incubated with bilirubin $(0.343 \mathrm{mM}), \mathrm{L}-\alpha$-phosphatidylcholine $(0.75 \mathrm{mg} / \mathrm{ml})$, saccharolactone $(8.5 \mathrm{mM}), \mathrm{MgCl}_{2}(10 \mathrm{mM})$, and $\left[{ }^{14} \mathrm{C}\right] \operatorname{UDPGA}(5 \mathrm{mM})$ in a $0.5 \mathrm{M}$ Tris$\mathrm{HCl}$ medium ( $\mathrm{pH} \mathrm{7.4)} \mathrm{for} 60 \mathrm{~min}$ at $37^{\circ} \mathrm{C}$, in a final volume of $100 \mu \mathrm{l}$. The reaction was stopped with $100 \mu \mathrm{l}$ of $0.7 \mathrm{M}$ glycine- $\mathrm{HCl}$ buffer ( $\mathrm{pH}$ 2.0). Radiolabeled bilirubin glucuronides were extracted into ethyl acetate $(1 \mathrm{ml})$, and the radioactivity in $300 \mu \mathrm{l}$ aliquots was measured using a liquid scintillation counter (Tri-Carb ${ }^{\circledR} 4530$; Packard, Meriden, CT).
PNP glucuronidation assay. A preincubated $\left(4^{\circ} \mathrm{C}\right.$ for $\left.30 \mathrm{~min}\right)$ mixture of human liver microsomes $(1 \mathrm{mg} / \mathrm{ml})$ with Triton X-100 $(0.025 \% \mathrm{vol} / \mathrm{vol})$ was incubated (final volume $200 \mu \mathrm{l})$ with PNP (360 $\mu \mathrm{M})$, EDTA $(40 \mu \mathrm{M}), \mathrm{MgCl}_{2}(10 \mathrm{mM})$, UDPGA $(2 \mathrm{mM})$ in a $0.1 \mathrm{M}$ Tris- $\mathrm{HCl}$ buffer $\left(\mathrm{pH} \mathrm{7.4)}\right.$ for $10 \mathrm{~min}$ at $37^{\circ} \mathrm{C}(31-33)$. The reaction was stopped by the addition of $2.8 \mathrm{ml}$ of $0.2 \mathrm{M}$ glycine- $\mathrm{NaOH}$ buffer $(\mathrm{pH}$ 10.4). Samples were centrifuged at 2,500 rpm for $30 \mathrm{~min}$, and UGT activity in the supernatant was measured colorimetrically from the disappearance of the substrate at $\lambda=400 \mathrm{~nm}$, using a spectrophotometer (DU ${ }^{\circledR}-50$; Beckman Instruments, Inc., Fullerton, CA).

Stable expression of human UGT1A1, UGT1A4, and UGT2B7. Culture media and conditions for the stable expression of human UGT1A1, UGT1A4, and UGT2B7 have been described previously (34-37). Briefly, the XbaI/XhoI and bamH1-XhoI fragments of human UGT1A1, UGT1A4, and UGT2B7 were subcloned into the respective corresponding sites of the mammalian expression vectors pcDNA3, pREP9, and pcDNA3.1. HK293 cells were transfected with the vectorUGT isozyme using the calcium phosphate transfection method (38).

Membrane preparations of human UGT1A1. Membrane preparations of human UGT1A1 were made by modification of methods described previously $(39,40)$.

\section{Results}

In vitro glucuronidation of $\mathrm{SN}-38$ in liver microsomes from normal rats and humans. The chromatograph obtained after in vitro glucuronidation of $\mathrm{SN}-38$ in human microsomes is illustrated in Fig. 2 A. Retention times for SN-38G, SN-38, and CPT were $\sim 3.5,9$, and $10 \mathrm{~min}$, respectively. Similar chromatographs were obtained using normal rat liver microsomes (data not shown). The peaks for the three compounds were confirmed using standards of SN-38G, SN-38, and CPT. The SN$38 \mathrm{G}$ peak was further confirmed by treatment with $1,000 \mathrm{U}$ of $\beta$-glucuronidase enzyme (from Escherichia coli, in $0.1 \mathrm{M}$ sodium phosphate buffer, $\mathrm{pH}$ 6.5), which resulted in the loss of the glucuronide (data not shown).

Lineweaver-Burk analysis of the data from normal human liver microsomes resulted in $K_{\mathrm{m}}$ and $V_{\max }$ values of $4.1 \mu \mathrm{M}$ and $40 \mathrm{pmol}$ of $\mathrm{SN}-38 \mathrm{G}$ formed $/ \mathrm{min} / \mathrm{mg}$ microsomal protein, respectively, for SN-38 UGT. To evaluate interindividual variations in rates of $\mathrm{SN}-38$ glucuronidating activity, individual microsomes from 25 human livers were screened for in vitro SN-38 glucuronidating activity. There was considerable variation in the rates of $\mathrm{SN}-38 \mathrm{G}$ formation among livers, with a range of $0.5-26 \mathrm{pmol}$ of $\mathrm{SN}-38 \mathrm{G}$ formed $/ \mathrm{min} / \mathrm{mg}$ microsomal protein (mean \pm SD 13 \pm 9 , median 12$)$.

In vitro glucuronidation of $S N-38$ in liver microsomes from Gunn rats and $C N-I$ patients. Fig. 2, $B$ and $C$ represent the chromatographs obtained after in vitro incubation of SN-38 in microsomes from Gunn rats and CN-I patient 'a.' Microsomes from both Gunn rats and CN-I patient 'a' lacked SN-38 glucuronidating activity, as seen by the absence of the SN-38G peak observed in normal rats and humans. Livers from the other three CN-I patients also exhibited a lack of SN-38 glucuronidating activity.

In vitro glucuronidation of $A Z T$ and oxidation of $O^{6}-B G$ in liver microsomes from normal rats and humans, Gunn rats, and $C N-I$ patients. To ensure the viability and functional enzymatic activity of the microsomal preparations, the UGT activity toward another substrate, AZT, was investigated as a control. Intact in vitro formation of GAZT was observed in microsomes from all four sources. Under our HPLC conditions, UDPGA/UDP (coelution), GAZT, and AZT eluted at 

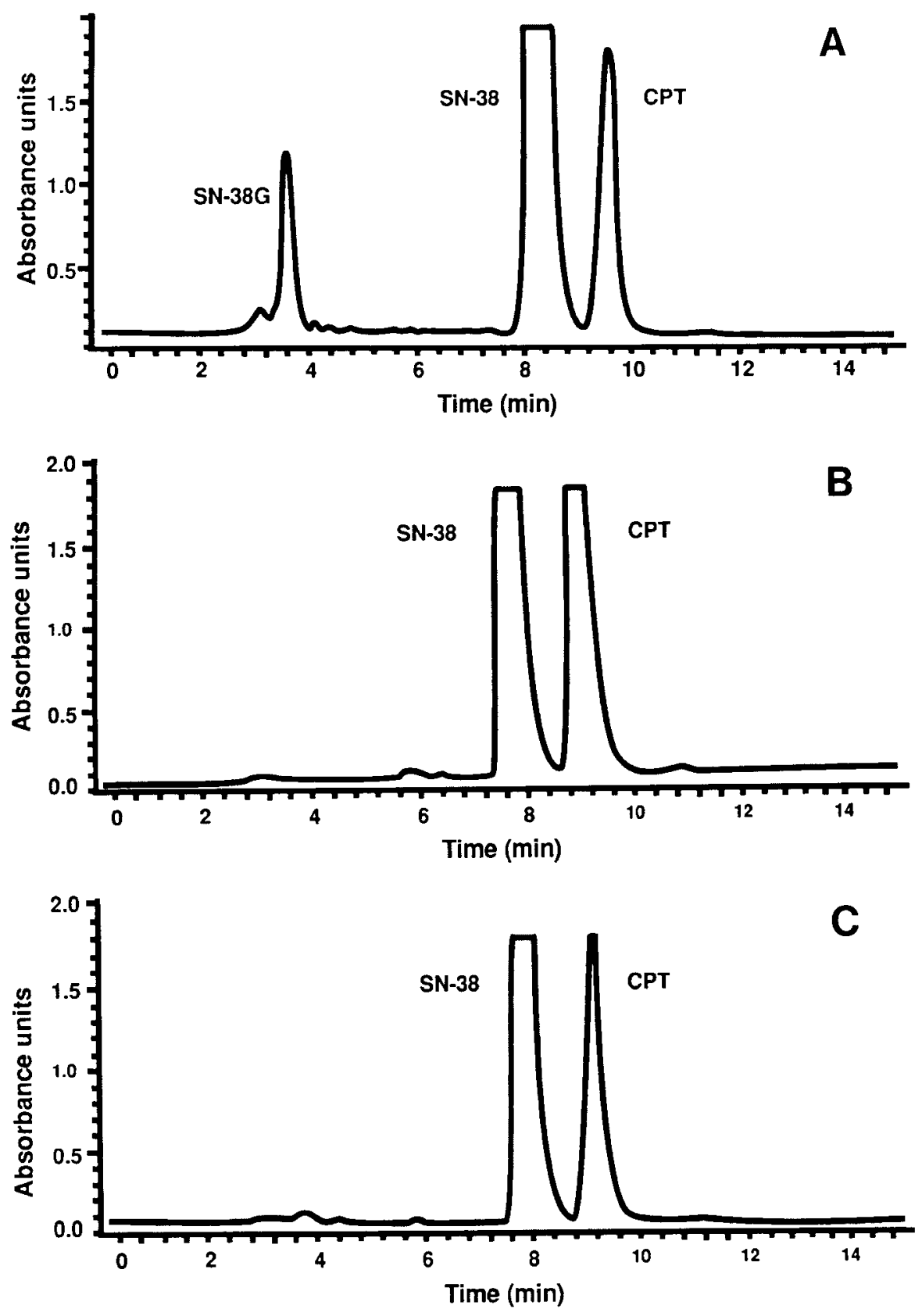

Figure 2. HPLC chromatographs obtained after in vitro glucuronidation of SN-38 in liver microsomes from normal humans, Gunn rats, and a patient with CN-I syndrome. SN-38 $(5 \mu \mathrm{M}), \mathrm{MgCl}_{2}(10 \mathrm{mM})$, and UDPGA $(5 \mathrm{mM})$ were incubated with microsomes $(1 \mathrm{mg} / \mathrm{ml})$ in a $0.1 \mathrm{M}$ Tris-buffer medium ( $\mathrm{pH} 7.4)$ for $30 \mathrm{~min}\left(37^{\circ} \mathrm{C}\right)$. After centrifugation, a $100 \mu \mathrm{l}$ aliquot of supernatant was injected into the column and eluted with $0.1 \mathrm{M} \mathrm{KH}_{2} \mathrm{PO}_{4}$ /acetonitrile (65: 35 ) at a constant flow rate of $0.8 \mathrm{ml} / \mathrm{min}$. $(A)$ Normal human liver microsomal pool $(n=4) ;(B)$ Gunn rat liver microsomal pool $(n=3) ;(C)$ liver microsomes from $\mathrm{CN}-\mathrm{I}$ patient 'a.' $\sim 2.6,5.7$, and $9.5 \mathrm{~min}$, respectively. These peaks appeared consistently after incubation of AZT with microsomes from normal rats and humans as well as in Gunn rats and CN-I patients (data not shown). The retention times obtained were confirmed using standard solutions of the respective chemicals. Intact oxidation of $O^{6}$-BG to $O^{6}$-benzyl-8-oxoguanine was also found in microsomes from all sources (data not shown).

Correlation studies with in vitro $S N-38$ glucuronidation versus bilirubin and PNP glucuronidation in human liver microsomes. The in vitro glucuronidation of $\mathrm{SN}-38$ in human liver microsomes was correlated with bilirubin glucuronidation, the results of which are illustrated in Fig. 3. There was a significant correlation between rates of formation of SN-38G and bilirubin mono- and diglucuronides (Pearson correlation coefficient, $r=0.89, P=0.001)$. In contrast, there was a poor association between SN-38G formation and PNP glucuronidation $(r=0.08, P=0.703)$. The rates of PNP glucuronidation were distributed evenly among subjects (mean \pm SEM 20.39 \pm 2.4, range $16.48-23.82 \mathrm{nmol} \mathrm{PNP}$ glucuronidated $/ \mathrm{min} / \mathrm{mg}$ protein).

SN-38 glucuronidation by cDNA-expressed isoforms of $U G T$. Bilirubin has been reported to be glucuronidated by two isozymes of UGT1: UGT1A1 and UGT1A4 (41). To determine which particular isozyme of UGT1 glucuronidates SN-38, HK293 cells transfected with cDNA encoding these isozymes, and a member of the UGT2 family (UGT2B7) were screened for SN-38 glucuronidating activity (Fig. 4). There was a lack of SN-38 glucuronidating activity in control HK293 cells and those expressing UGT1A4 and UGT2B7, up to protein 


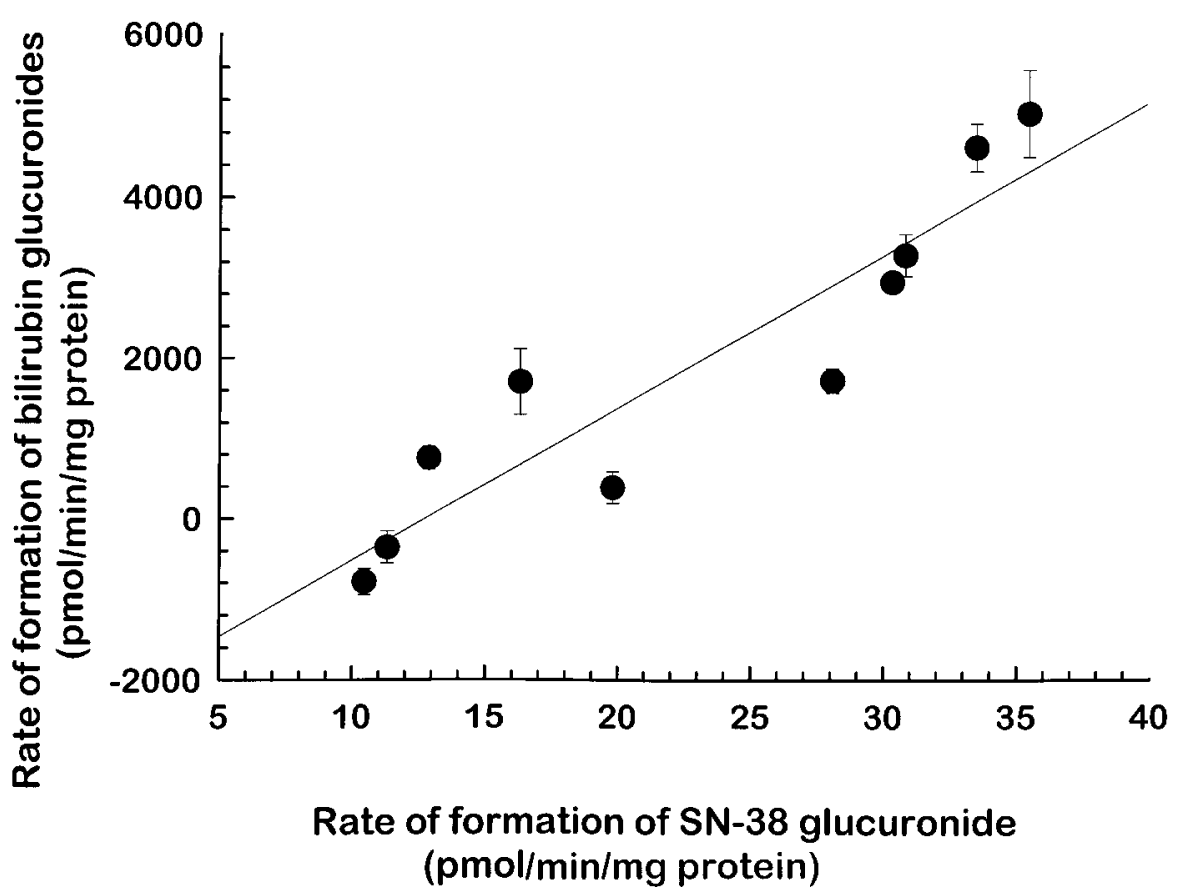

Figure 3. Correlation between in vitro SN38 and bilirubin glucuronidation in human liver microsomes. For bilirubin glucuronidation, microsomes $(0.5 \mathrm{mg} / \mathrm{ml})$ were incubated with bilirubin $(0.343 \mathrm{mM})$, phosphatidylcholine $(0.75 \mathrm{mg} / \mathrm{ml})$, saccharolactone $(8.5 \mathrm{mM}), \mathrm{MgCl}_{2}(10 \mathrm{mM})$, and $\left[{ }^{14} \mathrm{C}\right]$ UDPGA $(5 \mathrm{mM})$ in a $0.5 \mathrm{M}$ Tris- $\mathrm{HCl}$ medium ( $\mathrm{pH} 7.4$ ) for $1 \mathrm{~h}$ at $37^{\circ} \mathrm{C}$. The radiolabeled glucuronides were extracted into ethyl acetate, and the radioactivity was measured using a liquid scintillation counter. Each data point represents the mean of three separate determinations $\pm \operatorname{SEM}(r=0.89, P=0.001)$. concentrations of $2 \mathrm{mg} / \mathrm{ml}$. On the other hand, there was measurable SN-38G formation in cells expressing UGT1A1, which increased with increasing protein concentrations, indicating that this isoform catalyzes $\mathrm{SN}-38$ glucuronidation. This finding was further confirmed using membrane preparations from HK293 cells expressing UGT1A1, also shown in Fig. 4. The HK293 cells have been screened previously for control UGT activity using their respective substrates, bilirubin (UGT1A1 and UGT1A4) (35), tertiary amine substrates (UGT1A4) (36), and morphine (UGT2B7) (37).

\section{Discussion}

We have determined the role of UGT1A1 in the glucuronidation of SN-38, which was demonstrated by $(a)$ providing evidence for this isoform using specific models of UGT family deficiency, and $(b)$ identifying the specific isozyme among the UGT1 isozymes to be UGT1A1, using correlation studies and investigations with HK293 cells expressing specific UGT isozymes.

The absence of SN-38 glucuronidation in microsomes from

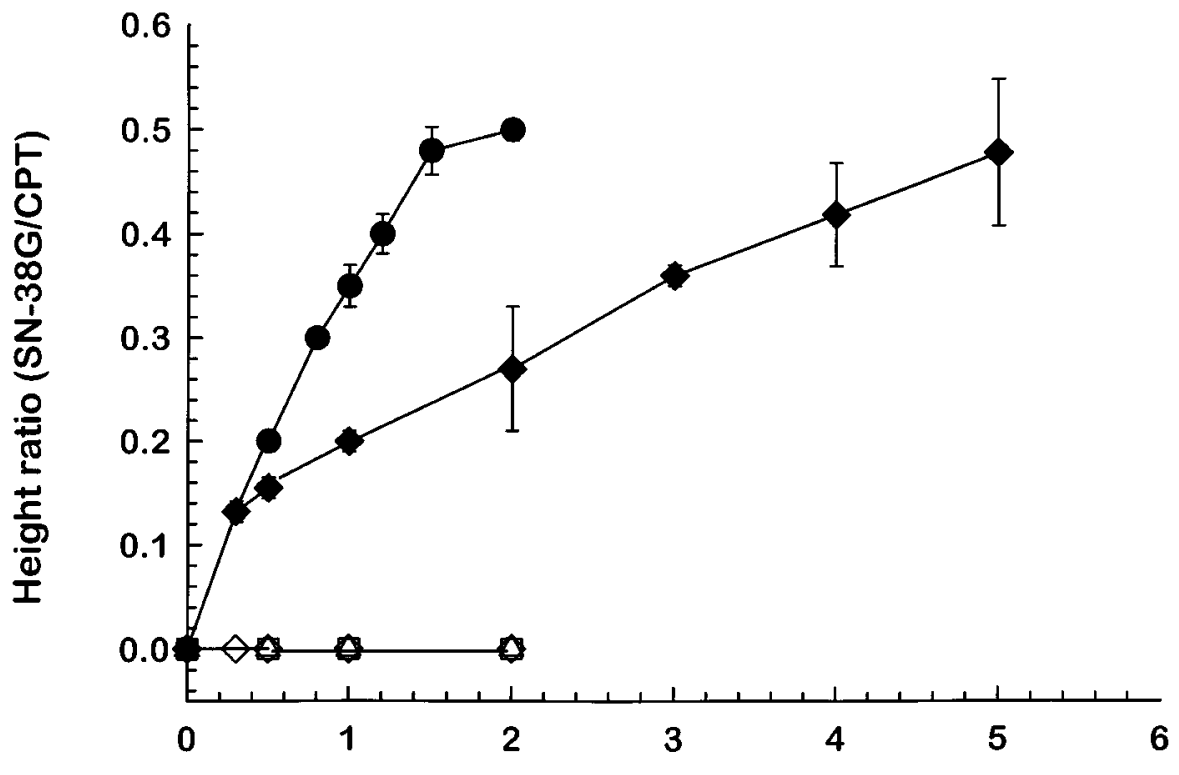

Protein Concentration (mg/ml)
Figure 4. In vitro glucuronidation of SN-38 by cDNA-expressed UGT isoforms. Cell lysates from HK293 cells expressing UGT1A1 ( ), UGT1A4 ( $\square$ ), and UGT2B7 enzymes $(\triangle)$, and membrane preparations of HK293 cells expressing UGT1A1 enzyme $(\bullet)$, were screened for SN-38 glucuronidating activity. HK293 cells $(\diamond)$ were used as control. Each data point represents the mean of three separate determinations \pm SEM. 
Gunn rats and CN-I patients (Fig. 2, $B$ and $C$ ) demonstrates the role of the UGT1 family in catalyzing SN-38 conjugation, and excludes UGT2 family isoforms as being significantly involved in SN-38 glucuronidation. Substrates of the UGT1 isoforms include bilirubin and phenols (18). Gunn rats have a deletion in the conserved portion of the UGT1 gene, resulting in a loss of UGT1 activity (20). The genetic lesion in Gunn rats parallels closely that observed in CN-I syndrome, where there may be variable substitutions of one or more amino acids in the coding region of bilirubin UGT (22). In our study, patient 'a' had a cytosine to thymine substitution in exon 4 of bilirubin/ phenol UGT, resulting in the substitution of phenylalanine (TTC) for a serine residue (TCC) (22). The exact molecular defects of UGT from patients 'b,' 'c,' and 'd' are unknown.

The oxidative capacity and AZT glucuronidating activity of the hepatic microsomes used in this study were evaluated as quality control measures of the microsome preparation method, using $O^{6}$-BG as a substrate for cytochrome P450 3A4 and $1 \mathrm{~A} 2$ enzymes (29) and AZT as a substrate for UGT in humans (31). AZT has been reported to be a substrate of the UGT2 isoform (31), although this finding requires further confirmation (16). The rate of formation of GAZT was more than twofold greater in human than in rat microsomes, in agreement with previous reports (27). AZT glucuronidating activity in Gunn rats was comparable to normal rats, and that in CN-I patients was comparable to normal humans. This is in agreement with other investigations where $\mathrm{CN}$-I patients could glucuronidate AZT, despite genetic deficiency in bilirubin UGT (42). $O^{6}$-BG oxidation was also comparable in UGT1-deficient microsomes and normal human and rat microsomes. These findings indicate that the failure to glucuronidate $\mathrm{SN}-38$ results specifically from the genetic defect in UGT1.

Additional studies correlating the rates of glucuronidation of SN-38 and other model compounds provided evidence of the specific isoform of UGT1 responsible. Planar phenols such as PNP and acetaminophen are glucuronidated predominantly by UGT1A6 $(43,44)$, although several other UGT isoforms such as UGT1A1, UGT1A4, and UGT2B15 have been reported to play a role in PNP glucuronidation in humans (35, 36). Our finding of inconsistency between SN-38 and PNP glucuronidation supports previous reports that acetaminophen clearance is a poor predictor of SN-38 glucuronidation (14). The similarity between SN-38 and bilirubin glucuronidation (Fig. 3) suggested that bilirubin UGT may also be glucuronidating SN-38. The cDNAs of two forms of human liver bilirubin UGT have been cloned (41), with reports of one isoform (B-UGT 1 or UGT1A1) being physiologically relevant in bilirubin glucuronidation (46). To resolve this in reference to SN-38, HK293 cells expressing these specific isozymes were screened for SN-38 UGT activity. In addition, cDNA-expressed activity of a member of the UGT2 family, UGT2B7, was also examined. Substrates of UGT2B7 include nonsteroidal antiinflammatory agents such as naproxen and ibuprofen (47), and opioids such as morphine (37). These experiments resulted in a clear demonstration of the role of UGT1A1 in glucuronidating SN-38 (Fig. 4), and excluded UGT1A4 (and UGT2B7). The high correlation between SN-38 and bilirubin glucuronidation suggests that UGT1A1 is the isoform involved in $\mathrm{SN}-38$ glucuronidation.

The considerable interliver variability in rates of $\mathrm{SN}-38$ glucuronidation in human liver microsomes indicates a possible polymorphic behavior of UGT1A1 enzyme. We do not rule out the contribution of other factors in this variability, such as variations in liver harvesting procedures, and postharvest degradation of UGT activity during transport/storage. However, this contribution might be minimal, if it exists at all, as the rates of PNP glucuronidation were evenly distributed among these liver microsomes.

The clinical implications of the role of UGT1A1 in the metabolism of CPT-11's active metabolite are far-reaching. SN-38 glucuronidation rates were $\sim 80$-100-fold less than corresponding bilirubin glucuronidation rates, suggesting that there is a marginal capacity that could be subjected to pharmacological manipulation. Coadministration of irinotecan with drugs that are substrates, inhibitors, or activators of the same isoform of UGT may result in interactions leading to altered antitumor activity and toxicity of irinotecan. For example, in a recent study, irinotecan administration in rats pretreated with valproic acid and phenobarbital resulted in an inhibition and induction of SN-38G formation, respectively (48).

A major consequence of our findings is the demonstration of a genetic role in the metabolism of irinotecan, as two models that are genetically deficient in bilirubin UGT activity also lacked SN-38 glucuronidating activity. This implies a genetic predisposition to the gastrointestinal toxicity exhibited by patients treated with irinotecan. Gilbert's syndrome is another inherited disorder characterized by abnormal bilirubin glucuronidating activity (49). Our results indicate that patients with this syndrome may be at an increased risk of irinotecaninduced gastrointestinal toxicity. A recent report of two patients with Gilbert's syndrome who experienced severe diarrhea and neutropenia after irinotecan treatment (50) provides further evidence for our finding. Metabolism of drugs such as acetaminophen (51) and tolbutamide (52) has been reported to be altered in Gilbert's disease. This syndrome is accompanied by a mild, chronic hyperbilirubinemia that is often benign and clinically inconsequential (53). The specific molecular defect in Gilbert's syndrome is the presence of an A(TA $)_{7}$ TAA fragment in the UGT1A1 promoter, instead of the $\mathrm{A}(\mathrm{TA})_{6}$ TAA segment in normal individuals $(54,55)$. There appears to be a wide variation in the incidences of Gilbert's syndrome, ranging from $0.5-15 \%$ in various groups. Higher incidences (up to $19 \%$ ) of the homozygous A(TA) 7 TAA genotype have been reported recently in an Inuit population living in the Eastern Canadian Arctic (56).

An in vitro study investigating UGT1A1 activity and genotype in human liver microsomes is in progress. A phase I clinical trial is being initiated to investigate the relationship between UGT1A1 genotype/phenotype and gastrointestinal toxicity of irinotecan. Other possible substrates of UGT1A1 are also being evaluated as phenotypic probes of this enzyme activity. This could lead to a more appropriate, individualized dosing of irinotecan based on UGT1A1 genotype and/or phenotype, which would be a major advance in the efforts to improve the therapeutic index of irinotecan. Association of UGT1A1 activity with SN-38G exposure and SN-38 biliary index could not be investigated in earlier clinical studies (13) due to the lack of information on the specific role of UGT1A1 in SN-38 glucuronidation, and the lack of availability of a screening test for UGT1A1 polymorphism.

We conclude that $(a)$ UGT1A1 enzyme catalyzes the glucuronidation of SN-38, which is the active metabolite of irinotecan, (b) SN-38 glucuronidation may be subjected to pharmacological manipulation to improve the therapeutic index of 
irinotecan, and $(c)$ this finding is particularly relevant to populations with partial UGT1A1 activity, such as those with Gilbert's syndrome.

\section{Acknowledgments}

The authors would like to thank Jerry Collins (Food and Drug Administration Center for Drug Evaluation and Research, Rockville, MD) for the supply of human liver microsomes, Kiyoshi Terada (Yakult Honsha Co., Ltd.) for the gift of CPT and SN-38, and Drs. R.K. Verbeeck (Catholic University of Louvain, Belgium), D.A. Kelly, and A.J. Strain (Children's Hospital and Queen Elizabeth Hospital, Birmingham, UK) for the provision of livers from CN-I patients 'c' and 'd,' respectively. The technical assistance of Kevin Kent is sincerely appreciated.

This study was supported in part by National Institutes of Health grants T32-GM-07019 and GM-26221.

\section{References}

1. Kunimoto, T., K. Nitta, T. Tanaka, N. Uehara, H. Baba, M. Takeuchi, T. Yokokura, S. Sawada, T. Miyasaka, and M. Mutai. 1987. Antitumor activity of inhibitor 7-ethyl-10-[4-(1-piperidino)-1-piperidino]-carbonyloxy-camptothecin, a novel water-soluble derivative of camptothecin against murine tumors. Cancer Res. 47:5944-5947.

2. Houghton, P.J., P.J. Cheshire, J.C. Hallman, M.C. Bissery, A. MathieuBoué, and J.A. Houghton. 1993. Therapeutic efficacy of the topoisomerase I inhibitor 7-ethyl-10-[4-(1-piperidino)-1-piperidino]-carbonyloxy-camptothecin against human tumor xenografts: lack of cross-resistance in vivo in tumors with acquired resistance to the topoisomerase inhibitor 9-dimethylaminomethyl-10hydroxycamptothecin. Cancer Res. 53:2823-2829.

3. Vassal, G., M.J. Terrier-Lacombe, M.C. Bissery, A.M. Vénuat, F. Gyergyay, J. Bénard, J. Morizet, I. Boland, P. Ardouin, B. Bressac-de-Paillerets, and A. Gouyette. 1996. Therapeutic activity of CPT-11, a DNA-topoisomerase I inhibitor, against peripheral primitive neuroectodermal tumour and neuroblastoma xenografts. Br. J. Cancer. 74:537-545.

4. Rothenberg, M.L., J.R. Eckardt, J.G. Kuhn, H.A. Burris III, J. Nelson, S.G. Hilsenbeck, G.I. Rodriguez, A.M. Thurman, L.S. Smith, S.G. Eckardt, et al. 1996. Phase II trial of irinotecan in patients with progressive or rapidly recurrent colorectal cancer. J. Clin. Oncol. 14:1128-1135.

5. Kawato, Y., H. Nagata, T. Furuta, and T. Yokokura. 1991. Intracellular roles of SN-38, a metabolite of the camptothecin derivative CPT-11, in the antitumor effect of CPT-11. Cancer Res. 51:4187-4191.

6. Kawato, Y., M. Aonuma, K. Matsumoto, and K. Sato. 1991. Production of SN-38, a main metabolite of the camptothecin derivative CPT-11, and its species and tissue specificities. Yakubutsu Dotai. 6:899-907.

7. Rivory, L.P., J.-F. Riou, M.-C. Haaz, S. Sable, M. Vuilhorgne, A. Commercon, S.M. Pond, and J. Robert. 1996. Identification and properties of a major plasma metabolite of irinotecan (CPT-11) isolated from the plasma of patients. Cancer Res. 56:3689-3694.

8. Haaz, M.C., L.P. Rivory, C. Riché, and J. Robert. 1997. Metabolism of irinotecan (CPT-11) by human hepatic microsomes: participation of cytochrome P-450 3A (CYP3A) and drug interactions. Proc. Am. Assoc. Cancer Res. 38:17. (Abstr.)

9. Atsumi, R., W. Suzuki, and H. Hakusui. 1991. Identification of the metabolites of irinotecan, a new derivative of camptothecin, in rat bile and its biliary excretion. Xenobiotica. 21:1159-1169.

10. Kaneda, N., H. Nagata, T. Furuta, and T. Yokokura. 1990. Metabolism and pharmacokinetics of camptothecin analogue CPT-11 in the mouse. Cancer Res. 50:1715-1720.

11. Takasuna, K., Y. Kasai, Y. Kitano, K. Mori, K. Kakihata, M. Hirohashi, and M. Nomura. 1995. Study of mechanisms of diarrhea induced by the new anticancer agent drug irinotecan hydrochloride (CPT-11). Folia Pharmacol. Jpn. 105:447-460.

12. Araki, E., M. Ishikawa, M. Ligo, T. Koide, M. Itabashi, and A. Hoshi. 1993. Relationship between development of diarrhea and the concentration of SN-38, an active metabolite of CPT-11, in the intestine and the blood plasma of athymic mice following intraperitoneal administration of CPT-11.Jpn. J. Cancer Res. 84:697-702.

13. Gupta, E., T.M. Lestingi, R. Mick, J. Ramirez, E.E. Vokes, and M.J. Ratain. 1994. Metabolic fate of irinotecan in humans: correlation of glucuronidation with diarrhea. Cancer Res. 54:3723-3725.

14. Gupta, E., R. Mick, J. Ramirez, X. Wang, T. Lestingi, E.E. Vokes, and M.J. Ratain. 1997. Pharmacokinetics and pharmacodynamic evaluation of the topoisomerase inhibitor irinotecan in cancer patients. J. Clin. Oncol. 15:15021510.
15. Tephly, T., M. Green, J. Puig, and Y. Irshaid. 1988. Endogenous substrates for UDP-glucuronosyltransferases. Xenobiotica. 18:1201-1210.

16. Burchell, B., C.H. Brierly, and D. Rance. 1995. Specificity of human UDP-glucuronosyltransferases and xenobiotic glucuronidation. Life Sci. 57: 1819-1831.

17. Clarke, D.J., and B. Burchell. 1994. The uridine diphosphate glucuronosyltransferase multigene family: function and regulation. In Handbook of Experimental Pharmacology. F.C. Kaufmann, editor. Springer-Verlag, Berlin/ Heidelberg. 112:3-43.

18. Parkinson, A. 1996. Biotransformation of xenobiotics. In Casarett and Doull's Toxicology: The Basic Science of Poisons. C.D. Klaassen, editor. McGraw-Hill Inc., New York. 113-186.

19. Gunn, C.H. 1938. Hereditary acholuric jaundice in a new mutant strain of rats. J. Hered. 29:137-139.

20. Roy Chowdhury, J., R. Kondapalli, and N. Roy Chowdhury. 1993. Gunn rat: a model for inherited deficiency of bilirubin glucuronidation. Adv. Vet. Sci. Comp. Med. 37:149-173.

21. Crigler, J.F., and V.A. Najjar. 1952. Congenital familial nonhemolytic jaundice with kernicterus. Pediatrics. 10:169-180.

22. Bosma, P.J., J.R. Chowdhury, T.-J. Huang, P. Lahiri, R.P. Elferink, H.H. Van Es, M. Lederstein, P.F. Whitington, P.L. Jansen, and N.R Chowdhury. 1992. Mechanisms of inherited deficiencies of multiple UDP-glucuronosyltransferase isoforms in two patients with Crigler-Najjar syndrome, type I. FASEB (Fed. Am. Soc. Exp. Biol.) J. 6:2859-2863.

23. Purba, H.S., J.L. Maggs, M.L.E. Orme, D.J. Back, and B.K. Park. 1987. The metabolism of $17 \alpha$-ethinyl-oestradiol by human liver microsomes: formation of catechol and chemically reactive metabolites. Br. J. Clin. Pharmacol. 23: $447-453$.

24. Bradford, M.M. 1976. A rapid and sensitive method for the quantitation of microgram quantities of protein utilizing the protein-dye binding. Anal. Biochem. 72:248-254.

25. Iyer, L., S.K. Roy, and M.J. Ratain. 1996. In vitro glucuronidation of $\mathrm{SN}-38$, the active metabolite of irinotecan (CPT-11) in human liver microsomes. Proc. Am. Soc. Clin. Oncol. 15:1589. (Abstr.)

26. York, J.L. 1986. Enzymes: classification, kinetics and control. In Textbook of Biochemistry with Clinical Correlations. T.M. Devlin, editor. Wiley Medical, New York/Chichester/Brisbane/Toronto/Singapore. 117-176.

27. Haumont, M., J. Magdalou, C. Lafaurie, J.-M. Ziegler, G. Siest, and J.-N. Colin. 1990. Phenobarbital inducible UDP-glucuronosyltransferase is responsible for glucuronidation of $3^{\prime}$-azido-3'-deoxythymidine: characterization of the enzyme in human and rat liver microsomes. Arch. Biochem. Biophys. 281:264270 .

28. Sim, S.M., D.J. Back, and A.M. Breckenridge. 1991. The effect of various drugs on the glucuronidation of zidovudine (azidothymidine; AZT) by human liver microsomes. Br. J. Clin. Pharmacol. 32:17-21.

29. Roy, S.K., K.R. Korzekwa, F.J. Gonzalez, R.C. Moschel, and M.E. Dolan. 1995. Human liver oxidative metabolism of $O^{6}$-benzylguanine. Biochem. Pharmacol. 50:1385-1389.

30. Matern, H., H. Heinemann, and S. Matern. 1994. Radioassay of UDPglucuronosyltransferase activities toward endogenous substrates using labeled UDP-glucuronic acid and an organic solvent extraction procedure. Anal. Biochem. 219:182-188.

31. Rajaonarison, J.F., B. Lacarelle, G. De Sousa, J. Catalin, and R. Rahmani. 1991. In vitro glucuronidation of $3^{\prime}$-azido-3'-deoxythymidine by human liver. Role of UDP-glucuronosyltransferase 2 form. Drug Metab. Dispos. 19: 809-815.

32. Matsui, M., and H.K. Watanabe. 1982. Developmental alteration of hepatic UDP-glucuronosyltransferase and sulphotransferase towards androsterone and 4-nitrophenol in Wistar rats. Biochem. J. 204:441-447.

33. Watanabe, K., B. Hoskins, and I.K. Ho. 1986. Selective inhibitory effect of organophosphates on UDP-glucuronosyltransferase activities in rat liver microsomes. Biochem. Pharmacol. 35:445-460.

34. Coffman, B.L., M.D. Green, C.D. King, and T.R. Tephly. 1995. Cloning and stable expression of a cDNA encoding a rat liver UDP-glucuronosyltransferase (UDP-glucuronosyltransferase 1.1) that catalyzes the glucuronidation of opioids and bilirubin. Mol. Pharmacol. 47:1101-1105.

35. King, C.D., M.D. Green, G.R. Rios, B.L. Coffman, I.S. Owens, W.P. Bishop, and T.R. Tephly. 1996. The glucuronidation of exogenous and endogenous compounds by stably expressed rat and human UDP-glucuronosyltransferase 1.1. Arch. Biochem. Biophys. 332:92-100.

36. Green, M.D., W.P. Bishop, and T.R. Tephly. 1995. Expressed human UGT1.4 protein catalyzes the formation of quaternary ammonium-linked glucuronides. Drug Metab. Dispos. 23:299-302.

37. Coffman, B.L., G.R. Rios, C.D. King, and T.R. Tephly. 1997. Human UGT2B7 catalyzes morphine glucuronidation. Drug Metab. Dispos. 25:1-4.

38. Chen, C., and H. Okayama. 1988. Calcium phosphate-mediated gene transfer: a highly efficient system for stably transforming cells with plasmid DNA. Biotechniques. 6:632-638.

39. Battaglia, E., S. Senay, R. Fournel-Gigleux, G. Herber, G. Siest, and J. Magdalou. 1994. The chemical modification of human liver UDP-glucuronosyltransferase UGT1*6 reveals the involvement of a carboxyl group in catalysis. FEBS (Fed. Eur. Biochem. Soc.) Lett. 346:146-150. 
40. King, C.D., G.R. Rios, M.D. Green, P.I. MacKenzie, and T.R. Tephly. 1997. Comparison of stably expressed rat UGT1.1 and UGT2B1 in the glucuronidation of opioid compounds. Drug Metab. Dispos. 25:251-255.

41. Ritter, J.K., J.M. Crawford, and I.S. Owens. 1991. Cloning of two human liver bilirubin UGT cDNAs with expression in COS-1 cells. J. Biol. Chem. 266: 1043-1047.

42. Herber, R., J. Magdalou, M. Haumont, R. Bidault, H. Van Es, and G. Siest. 1992. Glucuronidation of 3'-azido-3'-deoxythymidine in human liver microsomes: enzyme inhibition by drugs and steroid hormones. Biochim. Biophys. Acta. 1139:20-24.

43. Burchell, B., D.W. Nebert, D.R. Nelson, K.W. Bock, T. Iyanagi, P.L.M. Jansen, D. Lancet, G.J. Mulder, J. Roy Chowdhury, G. Siest, et al. 1991. The UDP glucuronosyltransferase gene superfamily: suggested nomenclature based on evolutionary divergence. DNA Cell Biol. 10:487-494.

44. Harding, D., S. Fournel-Gigleux, M.R. Jackson, and B. Burchell. 1988. Cloning and substrate specificity of a human phenol UDP-glucuronosyltransferase expressed in COS-7 cells. Proc. Natl. Acad. Sci. USA. 85:8381-8385.

45. Green, M.D., E.M. Oturu, and T.R. Tephly. 1994. Stable expression of a human liver UDP-glucuronosyltransferase (UGT2B15) with activity toward steroid and xenobiotic substrates. Drug Metab. Dispos. 22:799-805.

46. Bosma, P.J., J. Seppen, B. Goldhoorn, C. Bakker, R.P.J. Oude Elferink, J. Roy Chowdhury, N. Roy Chowdhury, and P.L.M. Jansen. 1994. Bilirubin UDP-glucuronosyltransferase 1 is the only relevant bilirubin glucuronidating isoform in man. J. Biol. Chem. 269:17960-17964.

47. Jin, C., J.O. Miners, K.J. Lillywhite, and P.I. Mackenzie. 1993. Complementary deoxyribonucleic acid cloning and expression of a human liver uridine diphosphate-glucuronosyltransferase glucuronidating carboxylic acid-containing drugs. J. Pharmacol. Exp. Ther. 264:475-479.
48. Gupta, E., X. Wang, J. Ramirez, and M.J. Ratain. 1997. Modulation of glucuronidation of SN-38, the active metabolite of irinotecan, by valproic acid and phenobarbital. Cancer Chemother. Pharmacol. 39:440-444.

49. Gilbert, A., and P. Lereboullet. 1901. La cholémie simple familiale. $\mathrm{La}$ Semaine Medicale. 21:241-243.

50. Wasserman, E., A. Myara, D. Paumier, P. Herait, L. Awad, J.L. Misset, and E. Cvitkovic. 1997. Baseline bilirubin (bil) and its transient early increase predicts likelihood of severe neutropenia and diarrhea in CPT-11-based chemotherapy. Proc. Am. Soc. Oncol. 16:219a. (Abstr.)

51. de Morais, S.M.F., J.P. Uetrecht, and P.G. Wells. 1992. Decreased glucuronidation and increased bioactivation of acetaminophen in Gilbert's syndrome. Gastoenterology. 102:577-586.

52. Carulli, N., M.P. Leon, E. Mauro, F. Manenti, and A. Ferrari. 1976. Alteration of drug metabolism in Gilbert's syndrome. Gut. 17:581-587.

53. Fevery, J. 1981. Pathogenesis of Gilbert's syndrome. Eur. J. Clin. Invest. 11:417-418.

54. Monaghan, G., M. Ryan, R. Seddon, R. Hume, and B. Burchell. 1996. Genetic variation in bilirubin UDP-glucuronosyltransferase gene promoter and Gilbert's syndrome. Lancet. 347:578-581.

55. Bosma, P.J., J. Roy Chowdhury, C. Bakker, S. Gantla, A. Boer, B.A Oostra, D. Lindhout, G.N.J. Tytgat, P.L.M. Jansen, R.P.J.O. Elferink, and N.R Chowdhury. 1995. The genetic basis of the reduced expression of bilirubin UDP-glucuronosyltransferase 1 in Gilbert's syndrome. N. Engl. J. Med. 333: 1171-1175.

56. Monaghan, G., B. Foster, M. Jurima-Romet, R. Hume, and B. Burchell. 1997. UGT1*1 genotyping in a Canadian Inuit population. Pharmacogenetics. 7 : 153-156. 